\title{
Chronic Pulmonary Aspergillosis and Type 2 Diabetes Mellitus Complicating Granulomatosis with Polyangiitis in an Adult Saudi Male: A Case Report
}

\author{
Safwat Eldaabossi ${ }^{1,2}$ \\ Mustafa Saad' \\ Mohammed Alabdullah' \\ Amgad Awad 1,2 \\ Hussain Alquraini' \\ Ghada Moumneh' \\ Ali Mansour' \\ 'Almoosa Specialist Hospital, Al Ahsa, \\ Saudi Arabia; ${ }^{2}$ Al Azhar Faculty of \\ Medicine, Al Azhar University, Cairo, \\ Egypt
}

Correspondence: Safwat Eldaabossi

Email safwatchest@gmail.com
Background: Granulomatosis with polyangiitis (GPA) is an extremely rare autoimmune, necrotizing granulomatous disease of unknown etiology affecting small and medium-sized blood vessels. Chronic pulmonary aspergillosis (CPA) is a rare fungal infection with high morbidity and mortality that usually affects immunocompetent or mildly immunosuppressed patients with underlying respiratory disease. Antifungal agents (voriconazole, itraconazole) are the mainstay of therapy. Intravenous drug therapy (amphotericin B or an echinocandin), alone or in combination with azoles, is the last resort in special situations such as azole failure, resistance, or severe disease. Sometimes CPA and GPA coexist and are difficult to distinguish due to the nonspecific symptoms and similarity of clinical and radiological features, so a high degree of suspicion is required to make the correct diagnosis.

Case Presentation: We reported that a 28 -year-old man from Saudi Arabia was diagnosed with GPA. The patient had been complaining of cough, fatigue, polyarthralgia and red eyes for 40 days before he was admitted to our hospital. The diagnosis of GPA was confirmed by clinical and radiological examinations and a pathological report of a lung biopsy, and he was treated with immunosuppressive drugs. The patient's condition was complicated by chronic pulmonary aspergillosis and type 2 diabetes mellitus. Initial treatments included systemic glucocorticoids, methotrexate, followed by rituximab and voriconazole, finally intravenous cyclophosphamide and amphotericin $\mathrm{B}$, with no complete remission. The thoracic surgical team postponed surgical debridement of the significant cavitary lung lesions until the active fungal infection could be brought under control.

Conclusion: The clinical and radiological features of GPA are similar to those of pulmonary tuberculosis, chronic pulmonary aspergillosis, and lung cancer. The lack of clear clinical symptoms of GPA requires a high degree of suspicion for early diagnosis. This case illustrates the dilemma of diagnosis and treatment of GPA and superimposed fungal infection. Secondary infection, particularly fungal infection, must be considered when GPA cannot be controlled with an immunosuppressant.

Keywords: granulomatous with polyangiitis, pulmonary aspergillosis, lung abscess, vasculitis

\section{Background}

Granulomatosis with polyangiitis (GPA) (formerly known as Wegener's granulomatosis) is an extremely rare autoimmune, necrotizing granulomatous disease of unknown etiology that affects small and medium-sized blood vessels in multiple organs, but most 
commonly the upper respiratory tract, lungs (more than $90 \%$ ), and kidneys, but also the skin, eyes, muscles, and nervous system. ${ }^{1,2}$ Treatment of GPA depends on its severity and whether it has resulted in organ damage. In patients with active, non-severe GPA (GPA without life- or organthreatening manifestations), start induction treatment with methotrexate and glucocorticoids. In patients with active, severe GPA, treatment with rituximab versus cyclophosphamide is recommended to induce remission and may improve survival by $20 \%$ to $80 \%$. Rituximab may be preferable in cases of hepatic or renal dysfunction or recurrent relapses during treatment with methotrexate.,

Chronic pulmonary aspergillosis (CPA) is a rare fungal infection with high morbidity and mortality. CPA usually occurs in patients with underlying pulmonary diseases such as previous tuberculosis, COPD, sarcoidosis, or lung cancer. The diagnosis of CPA depends on the presence of compatible clinical symptoms and radiologic findings with a positive serum Aspergillus precipitin test or isolation of an Aspergillus species from respiratory specimens (ie, sputum or bronchial aspirate fluid). Oral triazole therapy for CPA is now considered standard treatment. Oral voriconazole is also effective in CPA and has been acceptably tolerated in several studies as first-line therapy or after itraconazole (either due to failure or intolerance). ${ }^{5}$

Sometimes CPA and GPA coexist and are difficult to distinguish due to the nonspecific symptoms and similarities in clinical and radiological features, so a high degree of suspicion is required to make the correct diagnosis. ${ }^{6}$

The prevalence of fungal infections in Saudi Arabia is not known because there are no data on the burden of fungal diseases in Saudi Arabia. ${ }^{7}$ The prevalence of CPA has been estimated to be 3.4/100,000 in Saudi Arabia. ${ }^{8}$ There are fewer cases of CPA associated with superimposed pulmonary Aspergillus infections, especially in
Saudi Arabia. We report this case from eastern Saudi Arabia, a case of GPA with multiple pulmonary nodules that had developed into multiple cavitary lesions and were refined by chronic pulmonary aspergillosis.

\section{Case Presentation}

In this report, we describe a 28 -year-old Saudi man who was previously healthy. His medical history dates back to January 2020, when he had a 40-day dry cough, fever, fatigue, and red eyes, as well as abnormal chest x-rays. He smoked 10 packs a year. In August 2019, he underwent a lumbar laminectomy for left sciatica. Prior to admission, he had dry cough, red eyes, blurred vision, arthralgia in both knees, and low back pain. His chest X-ray showed an isolated pulmonary nodule in the right upper lobe, so he was referred to the pulmonary clinic (Figure 1A).

Since pulmonary tuberculosis is common in our area, we performed outpatient tuberculosis screening by sputum analysis for acid fast bacteria by smear and PCR. The result of the test was negative. A second chest X-ray 2 weeks later revealed bilateral pulmonary nodules, the largest in the right upper lobe.

He was admitted to another hospital, where an examination and flexible bronchoscopy with transbronchial biopsy of the right upper lobe was performed, which revealed no endoluminal lesion.

He denied fever, rash, numbness, headache, joint swelling, nasal congestion or discharge, or change in urine color. His examination revealed a body mass index of $30.5 \mathrm{~kg} / \mathrm{m} 2$, a temperature of $37.0^{*} \mathrm{C}$, a blood pressure of $130 / 80 \mathrm{mmHg}$, a heart rate of 110 beats per minute, and an oxygen saturation of $95 \%$. His eyes were red bilaterally, without discharge. We found no nasal polyps or deformities. The chest was auscultated bilaterally with equal air entry. His abdomen was neither tender nor distended and

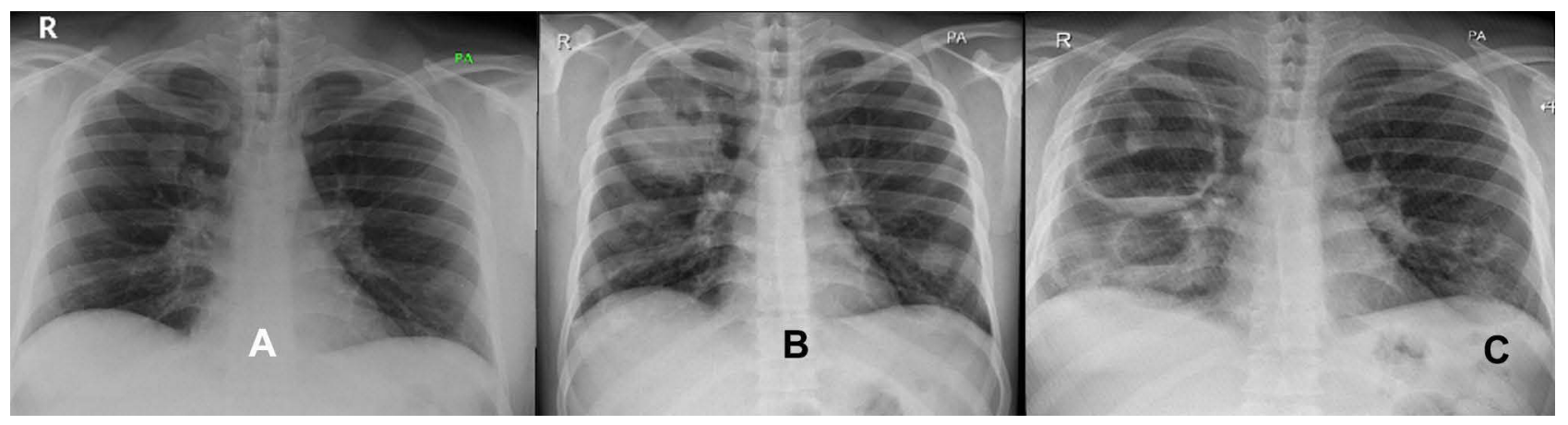

Figure I (A) Radiograph of the right upper lobe nodal consolidation with internal cyst formation. (B) Radiograph of right upper lobe cavitary mass like opacity with left lower lobe nodule. (C) Chest radiograph, multiple bilateral cavitary lesions with air-fluid levels, and the largest in the right upper lobe. 
there was no evidence of organomegaly. The patient had no accessible lymphadenopathy, rash, or arthritis. An ophthalmologist noted bilateral episcleritis.

Subsequent chest radiographs showed: enlargement of the right upper lobe nodule with formation of a cavitary massive opacity and enlargement of the left lower lobe nodule (Figure 1B).

An imaging examination on February 10, 2020 revealed multiple nodules of varying sizes on both sides of the chest and an enlarged lung mass in the right upper lobe with multiple mediastinal lymph nodes (Figure 2A and B). Infectious etiologies included bacterial infections, tuberculosis, and even mycoses. Sarcoidosis and granulomatosis with polyangiitis are other differential diagnoses. The possibility of a neoplastic process also cannot be ruled out. His investigations revealed microscopic haematuria and proteinuria and mild eosinophilia in the blood (Table 1).

He was admitted to our hospital on February 15, 2020, and a CT -guided lung biopsy was performed under local anesthesia without complications from the cavitary lesion of the right upper lobe (Figure $2 \mathrm{C}$ and $\mathrm{D}$ ). In addition to intravenous steroids (methylprednisolone 1), we started ambulatory steroids (prednisolone $50 \mathrm{mg}$ daily) and methotrexate $25 \mathrm{mg}$ every other week for five days.
Histopathology of the cavitary lesion of the right upper lobe revealed the following: The medium-sized vessels (arteries and veins) are typically absent due to inflammation and are replaced by scattered necrosis containing karyorrhectic neutrophils and eosinophils with fibrinoid necrosis (probable foci of leukocytoclastic vasculitis). The lesion consists of epithelioid-granulomatous collections of granulomas with occasional central small necrosis and multinucleated giant Langerhans cells. An overlap syndrome between GPA and eosinophilic granulomatosis with polyangiitis has been reported in the literature. Eosinophilic granulomatosis with polyangiitis was ruled out in this case based on the clinical history (no asthma, no atopy, and no significant peripheral eosinophilia) (Figure $3 \mathrm{~A}$ and $\mathrm{B}$ ).

His immunological profile showed positive results for antineutrophil cytoplasmic antibody (ANCA) C, rheumatoid factor, but negative results for $\mathrm{p}$-ANCA, antinuclear antibody (ANA) and anticyclic citrullinated antibody (CCA). As he has no children and is single, he refused cyclophosphamide.

He received a bolus of intravenous (IV) methylprednisolone three times a month for 3 months and oral prednisolone $50 \mathrm{mg}$ in combination with methotrexate $25 \mathrm{mg}$ once a week and sometimes mycophenolate

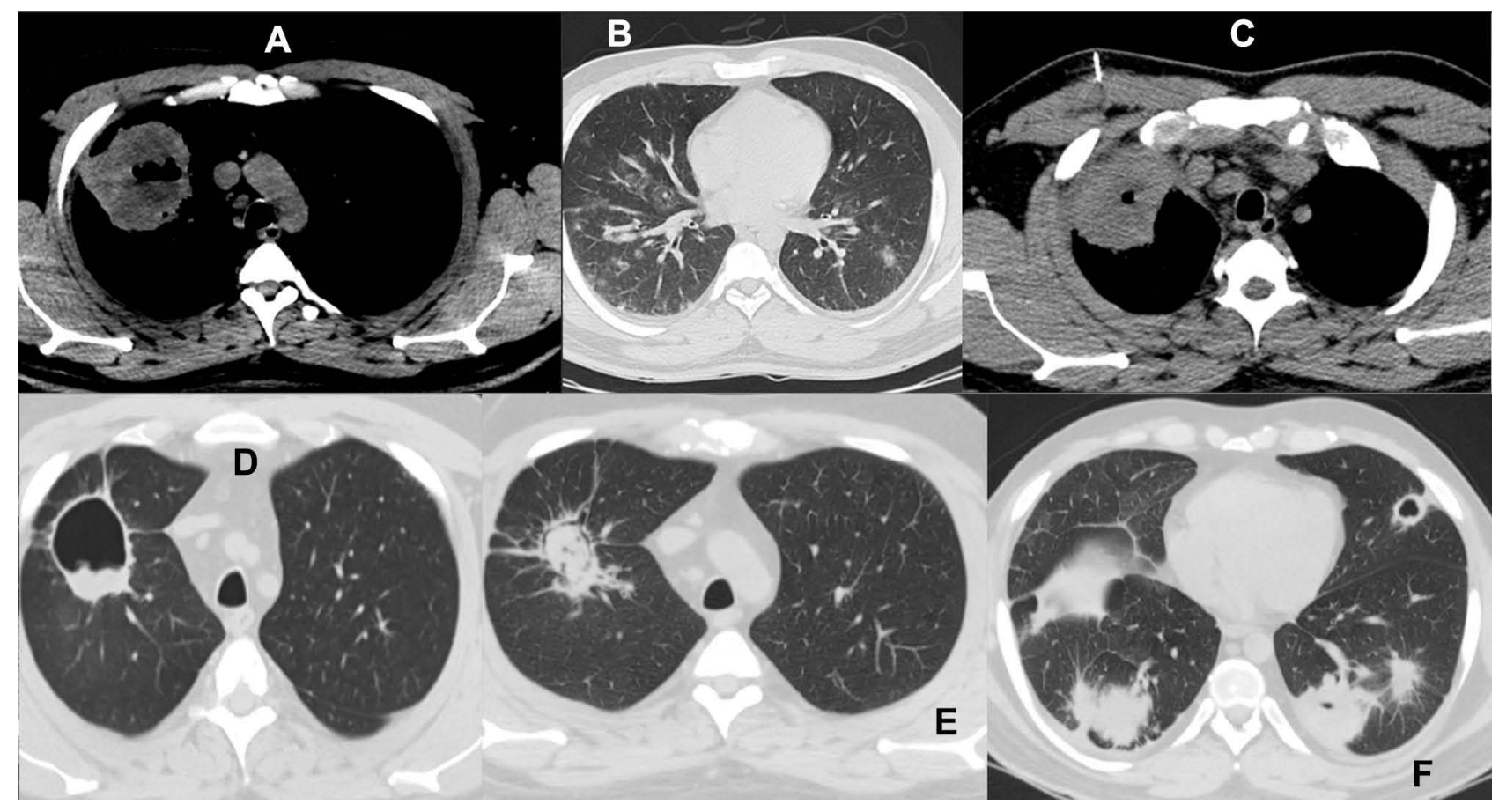

Figure 2 (A) CT thorax mediastinal window: right upper lobe mass like with central cavitation, $7.3 \times 7 \mathrm{~cm}$ in size. (B) CT thorax pulmonary window, multiple bilateral nodules show dominance of lower lobe with bilateral mild bronchial wall thickening. (C) CT thorax mediastinal window: CT-guided needle biopsy from the right upper lobe mass with central cavitation. (D) CT thorax pulmonary window. Cavitation of the right upper lobe. (E) CT thorax pulmonary window, October I/202I: Right upper lobe cavitary lesion measuring $3.8 \times 2.4 \mathrm{~cm}$, previously $3.8 \times 3 \times 2.8 \mathrm{~cm}$ with solid internal component and Crescent air sign, raising the possibility of underlying fungal ball. (F) CT thorax pulmonary window, October I/202I: showed bilateral cavitary lung lesions, one right lower lobe, one lingua and 2 left lower lobe. 
Table I Laboratory Results on Admission and Follow Up

\begin{tabular}{|c|c|c|c|c|c|c|}
\hline & February 2020 & September 2020 & November 2020 & March 202I & July 2021 & October 2021 \\
\hline Hemoglobin g/l & 13.1 & 13.5 & 13.2 & & & 12.9 \\
\hline Hematocrit & 36.6 & 38.7 & 38.9 & & & \\
\hline WBC Count $\times 109 / 1$ & 19.0 & 25.5 & 18.7 & 13.4 & & 22.2 \\
\hline RBC Count $\mid 012 / /$ & 4.97 & 5.23 & 5.26 & 5.6 & & 5.5 \\
\hline Platelets $\times 109 / 1$ & 345 & 433 & 458 & 490 & & 476 \\
\hline Rheumatoid Factor & Positive & & & & & \\
\hline ANA, Anti CCP & Negative & & & & & \\
\hline $\begin{array}{l}\text { Erythrocyte sedimentation } \\
\text { rate } \mathrm{mm} / \mathrm{h}\end{array}$ & 80 & 110 & 74 & 45 & & \\
\hline C-reactive protein, $\mathrm{mg} / \mathrm{l}$ & 80 & 350 & 180 & 34 & & 169 \\
\hline Transferees, Alanine & 43 & 67 & 69 & 22 & & 15 \\
\hline Transferees, Aspartate & 24 & 42 & 25 & 34 & & 13 \\
\hline HBAIc & 6 & 9.6 & 8.5 & 8 & 7 & \\
\hline C-ANCA & 1.20 & $\mathrm{I}: 20$ & $\mathrm{I}: 10$ & & & \\
\hline p-ANCA & Negative & Negative & Negative & & & \\
\hline Urine analysis Albumin & Trace & Trace & Nil & Nil & & \\
\hline WBCs urine & $2-5$ & $2-5$ & $2-5$ & $2-5$ & & $0-2$ \\
\hline RBCs urine & $20-25$ & $2-5$ & $2-5$ & $2-5$ & & $0-2$ \\
\hline $\begin{array}{l}\text { Sputum culture } \\
\text { Blood culture }\end{array}$ & Negative & Negative & Negative & Negative & & Negative \\
\hline Serum galactomannan & & Negative & Negative & & Negative & \\
\hline Sputum fungal culture & & $\begin{array}{l}\text { Positive for } \\
\text { aspergillus }\end{array}$ & $\begin{array}{l}\text { Positive for } \\
\text { aspergillus }\end{array}$ & Negative & & $\begin{array}{l}\text { Positive for } \\
\text { aspergillus }\end{array}$ \\
\hline $\begin{array}{l}\text { BALF galactomannan } \\
\text { Normal: }<0\end{array}$ & & & & & 8.3 & \\
\hline
\end{tabular}

mofetil (2 grams daily). After 3 months, his condition still had not improved clinically and radiologically, so rituximab 2 grams IV $500 \mathrm{mg}$ was given every 2 weeks for 4 doses, but he developed a severe allergic reaction after the fourth dose.

On September 17, 2020, in addition to fever, he developed increased cough, yellowish, sometimes bloody sputum, fatigue, and shortness of breath. He developed arthralgia and his eyes became red again. Clinically, he had a temperature of $39^{\circ} \mathrm{C}$, a pulse of 140 beats per minute, and a respiratory rate of 28 beats per minute.
Eyes were red, no lower limb edema, no rash, no arthritis Heart murmur was normal with no murmur. Dullness in the chest over both bases and distant air entry. A repeat chest $\mathrm{x}$-ray showed progression of dimensions of multiple cavities with air-fluid levels (Figure 1C). Blood and sputum cultures were negative for bacteria, but sputum cultures were positive for Aspergillus on 3 occasions. On many occasions, the serum galactomannan test was negative. His inflammatory markers were elevated, and COVID -19 was repeatedly negative. He was recently diagnosed with type 2 diabetes and was taking basal and normal 


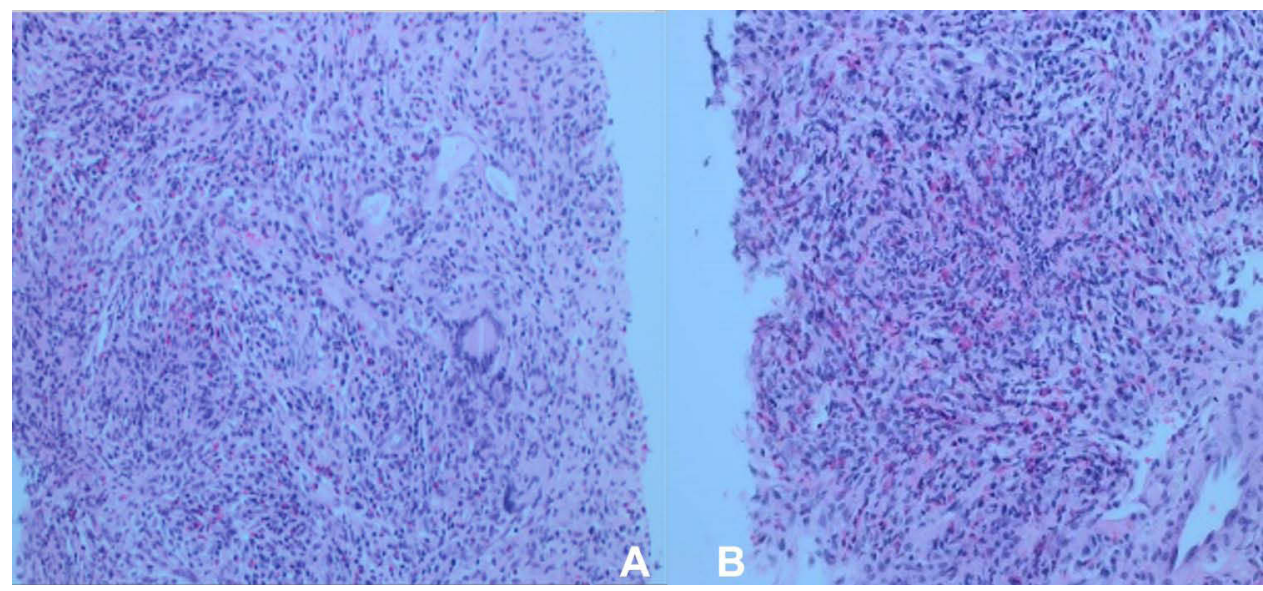

Figure 3 (A and B) Histopathological findings of lung biopsy of right upper lobe, medium-sized vessels (arteries and veins) are rarely seen and are probably destroyed by the inflammation and replaced by scattered relatively small necrosis consisting of degenerating karyorrhectic neutrophils with basophilia and eosinophils with fibrinoid necrosis (probable foci of leukocytoclastic vasculitis). 8, there are small epithelioid granulomatous collections with occasional central small necrosis, and few multinucleated giant Langerhans-type cells are noted.

insulin. Empirically, an intravenous antibiotic was initiated, but after the result of a culture, voriconazole $200 \mathrm{mg} / 8$ hours was administered intravenously for two weeks, followed by an oral dose.

During treatment, prednisolone $20 \mathrm{mg}$ daily and voriconazole $200 \mathrm{mg}$ thrice daily were given along with azathioprine $100 \mathrm{mg}$ pod daily and trimethoprim and sulfamethoxazole $480 \mathrm{mg}$ daily. He had purulent manifestations (cough and sputum with excessive sputum and fever, myalgia and fatigue with elevated inflammatory markers with negative sputum and blood cultures. Multiple courses of IV broad spectrum antibiotics were administered, each lasting three weeks, both inpatient and outpatient.

In March 2021, he was clinically stable with partial radiological improvement.

He developed bilateral cataracts and a bilateral catarcetomy was performed on 2 occasions under local anesthesia.

In June 2021, he developed an episode of purulent manifestations (cough and excessive sputum) with fever, myalgia and fatigue with an increase in inflammatory markers with negative sputum and blood cultures. Fungal culture in sputum was positive for Aspergillus fumigatus. Intravenous cyclophosphamide $500 \mathrm{mg}$ monthly for 3 months with intravenous amphotericin b (Ambisome) $200 \mathrm{mg}$ daily for 12 weeks. He began to become asymptomatic and improved clinically, radiologically, and laboratory. The oral steroid was reduced to $10 \mathrm{mg}$ prednisolone daily. With trimethoprim and prophylaxis for gastritis and osteoporosis.

After 12 weeks of treatment with IV Ambisome and restarting oral voriconazole (200 $\mathrm{mg}$ three times daily) without sufficient response, he was admitted to King Faisal Special Hospital in Riyadh and evaluated in July 2021 by flexible bronchoscope (BAL) and CT guided lung biopsy of the left lower lobe. BALF was negative for malignancy, viruses and fungi (by staining) but positive for galactomannan $(8,3)$. Serum galactomannan was negative (0.09).

The cardiothoracic surgeon, pulmonary rheumatology, and infectious disease team recommended continuing drug treatment for the fungal infection, as the patient had multiple lung cavity lesions, some of which had air fluid (active infection?), and reducing the oral corticosteroid to $10 \mathrm{mg}$ daily. Lung biopsy revealed nonspecific inflammation.

Follow-up CT on October 1, 2021 showed that the bilateral cavitary lung lesions had decreased in size and had markedly increased wall thickening (Figure 2E and F).

On October 26, 2021, he was reexamined at King Faisal Specialized Center by a multidisciplinary team. It was recommended to continue medical treatment and perform surgical intervention after ponding until the fungal infection is under control or complications occur.

\section{Discussion and Conclusions}

Granulomatosis with polyangiitis is a form of systemic vasculitis that results in necrotizing granulomatous vasculitis of the respiratory tract, kidneys, skin, peripheral nervous system, joints, and other organs. ${ }^{1-3}$ The incidence of GPA is about 10 cases per 1 million people and is slightly more common in men than in women, although there are no published data on the incidence of GPA in Saudi Arabia. ${ }^{4}$ The diagnosis of GPA depends on clinical, 
laboratory, radiological, and histopathological evaluation. GPA usually involves a triad of (a) upper respiratory tract (sinusitis, crusty rhinitis, saddle nose) and lower respiratory tract (pulmonary nodules, cavities, and alveolar haemorrhage), (b) systemic vasculitis, and (c) glomerulonephritis-ANCA-positive results (82 to 94\%) support but do not confirm the diagnosis of GPA. ${ }^{9}$ A biopsy of the affected organ is usually required to confirm the diagnosis and rule out infection or malignancy (scattered multinucleated giant cells, necrotizing granulomas with nonspecific inflammation and tissue necrosis accompanied by necrotizing vasculitis). ${ }^{2}$ In active, nonsevere GPA (GPA without life- or organ-threatening manifestations), induction therapy begins with methotrexate and glucocorticoids; methotrexate is equivalent but may be associated with a higher relapse rate. In patients with active, severe GPA, treatment with rituximab is recommended over cyclophosphamide for induction of remission. Rituximab may be preferable in cases of hepatic or renal dysfunction or recurrent relapse during treatment with methotrexate. Long-term therapy with trimethoprim/ sulfamethoxazole may prevent recurrent infections and prophylaxis of Pneumocystis carinii and induce remission of non-severe disease. ${ }^{3}$

Our patient had multiorgan involvement, including episcleritis, polyarthralgia, pulmonary nodules and cavities, microscopic hematuria, blood eosinophilia, positive c-ANCA and rheumatoid factor with elevated ESR and CRP. A lung biopsy revealed GPA and positive c-ANCA, ruling out the diagnosis of pulmonary tuberculosis and lung cancer. Initial treatment included systemic glucocorticoids, methotrexate, followed by rituximab and cyclophosphamide with a prophylactic dose of trimethoprim.

Infections, especially pulmonary infections, may also be a complication of immunosuppressive treatment. Symptoms of exacerbation of GPA or infection may be difficult to recognize in some clinical situations. The clinical picture, laboratory findings, and chest radiographs, especially high-resolution computed tomography (HRCT), are required for differentiation. The most complicated problem in the management of GPA patients is finding the right balance between the immunosuppression needed to treat the disease and minimizing the risk of bacterial infection. ${ }^{10}$

Aspergillosis is an infection of the lung caused by the genus Aspergillus (mostly Aspergillus fumigatus and Aspergillus flavus) and can be chronic or acute. Usually, chronic infection or colonization occurs in people with lung disease. There are several forms of chronic pulmonary aspergillosis, including chronic cavitary pulmonary aspergillosis and chronic fibrosing pulmonary aspergillosis, aspergillomas, and Aspergillus nodules. ${ }^{5}$ The prevalence of fungal infections in Saudi Arabia is not known because there are no data on the burden of fungal diseases in Saudi Arabia. ${ }^{7}$ The prevalence of CPA was estimated to be 3.4/100,000 in Saudi Arabia. ${ }^{8}$

The current criteria for establishing chronic pulmonary aspergillosis are (i) compatible clinical symptoms (fever, fatigue, sputum production, hemoptysis, or shortness of breath), (ii) compatible radiological findings (one large cavity or more than two small cavities, either with or without aspergilloma for 1-3 months), and (iii) a positive serum Aspergillus precipitin test or isolation of an Aspergillus species from respiratory specimens (ie, sputum, transtracheal aspirate, or bronchial aspirate fluid). ${ }^{5,11}$

The presence of a fungal ball strongly suggests aspergilloma as a manifestation of CPA. For a definitive diagnosis, microbiological confirmation is required to rule out other diagnoses such as tuberculosis and to determine the presence of fungus. Radiographs showing cavities may raise suspicion, but a positive Ig-G test for Aspergillus is required for confirmation. ${ }^{12,13}$ In a minority of patients with chronic pulmonary aspergillosis, cultures are positive for Aspergillus fumigatus, and serum galactomannan is usually negative. If the sputum culture is negative, bronchoscopy and bronchoalveolar lavage may be required. The BALF-galactomannan test has a sensitivity and specificity of about $38-50 \%$ and $87-100 \%$ for the diagnosis of CPA. ${ }^{14}$ Elevated inflammatory markers such as C-reactive protein and/or erythrocyte sedimentation rate are very common in patients with chronic pulmonary aspergillosis but are not specific. ${ }^{5,13}$ Current medical treatments for aggressive invasive aspergillosis include voriconazole and liposomal amphotericin $\mathrm{B}$ in combination with surgical debridement. ${ }^{5,13,15}$

Chronic cavitary pulmonary aspergillosis requires lifelong antifungal treatment. Oral triazole therapy for CPA is now considered standard treatment. Oral voriconazole (200 mg twice daily; may be increased to $300 \mathrm{mg}$ twice daily based on therapeutic drug monitoring) is also effective in CPA and has been acceptably tolerated in several studies as first-line therapy or after itraconazole $(200 \mathrm{mg}$ twice daily). Currently, the choice between the two agents depends on cost, availability, toxicity, and tolerability; therefore, they are used interchangeably as first-line therapy. 5,15 Intravenous drug therapy, alone or in 
combination with azoles, is the last resort in cases of clinical failure, pan-azole resistance, or drug toxicity, but can also be used in some situations with severe disease progression. Drugs administered intravenously include amphotericin B or an echinocandin. ${ }^{5,13}$

Surgery serves two important purposes for CPA patients: it can relieve symptoms that are disabling or lifethreatening, and it can even cure some patients if the localized disease does not respond to antifungal therapy. Due to the low prevalence of CPA and the significant number of health conditions that prevent surgery, the evidence on surgical intervention is limited to large case series compiled over long periods of time. , $^{46,17}$

This patient's condition was complicated by type 2 diabetes mellitus and chronic Aspergillus infection, which caused additional disease. This patient's diabetes was managed with basal insulin in combination with immunosuppression and antifungal therapy, so he was scheduled for surgical debridement of the two large cavities in the right upper and lower lobes, but the multidisciplinary team recommended continuing medical treatment and not performing surgery until the fungal infection was under control or if complications occurred.

GPA are extremely rare and the occurrence of aspergillosis is also extremely rare. Few cases of pulmonary Aspergillus infection have been reported as a complication of GPA. In both cases, pulmonary cavitations or nodules may occur. Detection of superimposed mycosis requires repeated sputum cultures for fungal detection and serum galactomannan (positive predictive value 25-62\%). In patients with diffuse lung involvement, bronchoalveolar lavage (BAL) is usually useful in diagnosing invasive pulmonary aspergillosis. Because of the risk of bleeding, transbronchial biopsies are not recommended. Histopathological examination is also required to diagnose invasive pulmonary aspergillosis..$^{11,13,16}$

Arfaj et al encountered 23 cases of GPA between 1990 and 2001 and reported that the lung was affected in $65.2 \%$ and the ANCA test was positive in $78.9 \%$. The findings of chest CT were pulmonary nodules in $80.0 \%$ and cavities in $(53.3 \%)$ patients. Lung biopsies showed chronic granulomatous inflammation with vasculitis and necrosis. $63 \%$ of patients received intravenous cyclophosphamide, and $10.5 \%$ required rituximab therapy. ${ }^{4} \mathrm{Al}-\mathrm{Harbi}$ et al investigated the etiology of lung granulomas (either transbronchial or endobronchial biopsies, CT -guided core biopsies, or surgical specimens) between 2005 and December 2013, and GPA was detected in 18 of 158 cases. ${ }^{18} \mathrm{He}$ et al reported: One patient with GPA had pulmonary mycosis (sputum cultures showed Candida albicans $3+$ and Aspergillus), which was successively treated with immunosuppressants and antifungal therapies. ${ }^{19}$ Richter et al examined the bronchoalveolar lavage fluid (BALF) of 33 patients with GPA to determine the predominant pathogens and reported the growth of mycoses in only one case. ${ }^{20}$ Invasive fungal disease is considered rare and prophylactic antifungal treatment should be considered in immunocompromised hosts. ${ }^{11}$ Yagi et al reported invasive pulmonary aspergillosis (IPA) due to A. lentulus in an adult diagnosed by bronchoscopy 3 weeks after starting systemic corticosteroid therapy for ANCA-associated vasculitis, which improved after treatment with the antifungal drug voriconazole. ${ }^{21}$ Seven of 157 patients with ANCAassociated vasculitis were found to have IPA. Two patients were classified as Wegener's granulomatosis and 5 as microscopic polyangiitis. Despite intensive antifungal therapy, only 3 patients survived. ${ }^{22}$ Le Thi Huong et $\mathrm{al}^{23}$ and Martens ${ }^{24}$ reported a case of pulmonary aspergilloma as a complication of Wegener's granulomatosis.

\section{What Makes Our Case Unique?}

The typical differential diagnosis for cavitary lesions of the lung is broad and may include infection (bacterial, tuberculosis, fungal), malignancy, septic embolism, infected cyst, necrotizing rheumatoid nodule, or GPA. Our case illustrates the dilemma in diagnosing and treating GPA and CPA in a patient who presents atypically, and this case is one of the few cases described in the literature as GPA with CPA.

\section{Conclusion}

The clinical and radiological features of GPA are similar to those of pulmonary tuberculosis, chronic pulmonary aspergillosis, and lung cancer. The lack of clear clinical symptoms of GPA requires a high degree of suspicion for early diagnosis. This case illustrates the dilemma of diagnosis and treatment of GPA and superimposed fungal infection. Secondary infection, particularly fungal infection, must be considered when GPA cannot be controlled with an immunosuppressant. Targeted diagnostic testing and appropriate early antifungal therapy should be instituted as soon as possible, minimizing immunosuppressive medication.

\section{Abbreviations}

ANCA, antineutrophil cytoplasmic antibody; ANA, antinuclear antibody; AIDS, Acquired immunodeficiency 
syndrome; BALF, bronchoalveolar lavage fluid; CPA, chronic pulmonary aspergillosis; RF, rheumatoid factor; ASP, Aspergillus; CT, computed tomography; GPA, granulomatosis with polyangiitis; IPA, invasive pulmonary aspergillosis; WBC, white blood count; MCV, mean corpuscular volume; C-S, culture and sensitivity; PCR, polymerase chain reaction; IV, intravenous; ESR, erythrocyte sedimentation rate; CRP, C-reactive protein; Anti CCA, anti-cyclic citrullinated antibodies; RBCS, red blood cells.

\section{Data Sharing Statement}

All data generated or analyzed during this study are included in this article.

\section{Ethics Declarations}

Ethics approval and consent to participate, Applicable open request.

Ethical approval was obtained from Review Board of Almoosa specialist hospital to publish the case details.

\section{Consent for Publication}

Written informed consent was given by the patient to publish the information in this case report. The consent form is available for review.

\section{Acknowledgments}

To the patient because he accepts to publish his condition, to Research center at Almoosa hospital, King Faisal specialized center, rheumatology, pulmonology thoracic surgery, and infectious diseases departments.

\section{Author Contributions}

All authors made a significant contribution to the work reported, whether that is in the conception, study design, execution, acquisition of data, analysis and interpretation, or in all these areas; took part in drafting, revising or critically reviewing the article; gave final approval of the version to be published; have agreed on the journal to which the article has been submitted; and agree to be accountable for all aspects of the work.

\section{Funding}

Almoosa Specialist Hospital research center will fund.

\section{Disclosure}

The authors declare that they have no competing interests in this work.

\section{References}

1. Singer O, McCune WJ. Update on maintenance therapy for granulomatosis with polyangiitis and microscopic polyangiitis. Curr Opin Rheumatol. 2017;29(3):248-253. doi:10.1097/BOR.00000000000 00382

2. Garlapati P, Qurie A. Granulomatosis with polyangiitis. In: StatPearls [Internet]. Treasure Island (FL): StatPearls Publishing; 2021. Available from https://www.ncbi.nlm.nih.gov/books/ NBK557827/. Accessed December 9, 2021.

3. Chung SA, Langford CA, Maz M, et al. 2021 American college of rheumatology/vasculitis foundation guideline for the management of antineutrophil cytoplasmic antibody-associated vasculitis. Arthritis Rheumatol. 2021;73(8):1366-1383. PMID: 34235894. doi:10.1002/ art.41773

4. Al Arfaj AS, Khalil N, Al Mogairen SA, Al Arfaj H, Al Khalaf A, Al Bedaiwi M. Pulmonary manifestations and radiographic findings in granulomatosis with polyangiitis patients in a university teaching hospital in Saudi Arabia. J Nat Sci Med. 2019;2(2). doi:10.4103/ JNSM.JNSM_38_18

5. Patterson TF, Thompson GR, Denning DW, et al. Practice guidelines for the diagnosis and management of aspergillosis: 2016 update by the infectious diseases society of America. Clin Infect Dis. 2016;63: e1-e60. doi:10.1093/cid/ciw326

6. Berden A, Göçeroglu A, Jayne D, et al. Diagnosis and management of ANCA associated vasculitis. BMJ. 2012;344:e26. doi:10.1136/ bmj.e26

7. Albarrag AM, Al-Abdely HM, Khalid NFA, Denning DW. Burden of serious fungal infections in Saudi Arabia. Available from: http://lifeworldwide.org/assets/uploads/files/ICAAC\%202014_\%20Fungal\% 20BurdenSaudi.pdf. Accessed June 5, 2017.

8. Wadi J, Denning DW. Burden of serious fungal infections in Jordan. J Fungi. 2018;4(1):15. PMID: 29371507; PMCID: PMC5872318. doi:10.3390/jof 4010015

9. Seo P, Stone JH. The antineutrophil cytoplasmic antibody-associated vasculitides. $A m \quad J \quad M e d . \quad 2004 ; 117(1): 39-50$. doi:10.1016/j. amjmed.2004.02.030

10. Dadoniene J, Pileckyte M, Baranauskaite A, et al. Clinical characteristics and long-term outcomes of 35 patients with Wegener's granulomatosis followed up at two rheumatology centers in Lithuania. Medicina. 2010;46:256-260. doi:10.3390/medicina46040035

11. Denning DW, Cadranel J, Beigelman-Aubry C, et al. Chronic pulmonary aspergillosis: rationale and clinical guidelines for diagnosis and management. Eur Respir J. 2016;47:45-68. doi:10.1183/ 13993003.00583-2015

12. Desai SR, Hedayati V, Patel K, Hansell DM. Chronic aspergillosis of the lungs: unravelling the terminology and radiology. Eur Radiol. 2015;25:3100-3107. doi:10.1007/s00330-015-3690-7

13. Denning DW, Page ID, Chakaya J, et al. Case definition of chronic pulmonary aspergillosis in resource-constrained settings. Emerg Infect Dis. 2018;24:e1-e13. doi:10.3201/eid2408.171312

14. Maghrabi F, Denning DW. The management of chronic pulmonary aspergillosis: the UK national aspergillosis centre approach. Curr Fungal Infect Rep. 2017;11(4):242-251. doi:10.1007/s12281-0170304-7

15. Aspergillosis | About; May 10, 2021. Available from: www.cdc.gov. Accessed June 1, 2021.

16. Barac A, Kosmidis C, Alastruey-Izquierdo A, et al. Chronic pulmonary aspergillosis update: a year in review. Med Mycol. 2019;57 (Supplement_2):S104-S109. doi:10.1093/mmy/myy070

17. Muniappan A, Tapias LF, Butala P, et al. Surgical therapy of pulmonary aspergillomas: a 30-year North American experience. Ann Thorac Surg. 2014;97:432-438. doi:10.1016/j.athoracsur.2013.10.050

18. Al-Harbi A, Al-Otaibi S, Abdulrahman A, et al. Lung granuloma: a clinicopathologic study of 158 cases. Ann Thorac Med. 2017;12:278-281. doi:10.4103/atm.ATM_1_17 
19. He Y, Liu J, Gao B. Wegener's granulomatosis with pulmonary fungal infection: a case report and brief review. $J$ Int Med Res. 2012;40:383-392. doi:10.1177/147323001204000141

20. Richter AG, Stockley RA, Harper L, Thickett DR. Pulmonary infection in Wegener granulomatosis and idiopathic pulmonary fibrosis. Thorax. 2009;64(8):692-697. doi:10.1136/thx.2008.110445

21. Yagi K, Ushikubo M, Maeshima A, et al. Invasive pulmonary aspergillosis due to Aspergillus lentulus in an adult patient: a case report and literature review. J Infect Chemother. 2019;25(7):547-551. doi:10.1016/j.jiac.2019.02.003

22. $\mathrm{Su} \mathrm{T}, \mathrm{Li} \mathrm{HC}$, Chen $\mathrm{M}$, et al. Invasive pulmonary aspergillosis in patients with antineutrophil cytoplasmic antibody associated vasculitis. $J$ Clin Rheumatol. 2009;15(8):380-382. PMID: 19279506. doi:10.1097/RHU.0b013e31819e67b1
23. Le Thi Huong D, Wechsler B, Chamuzeau JP, Bisson A, Godeau P. Pulmonary aspergilloma complicating Wegener's granulomatosis. Scand J Rheumatol. 1995;24(4):260. PMID: 7481595. doi:10.3109/ 03009749509100887

24. Martens J. Pulmonary aspergilloma, an unusual complication in Wegener's granulomatosis. Postgrad Med J. 1982;58(675):55-56. PMID: 7088764; PMCID: PMC2426230. doi:10.1136/pgmj.58.675.55

\section{Publish your work in this journal}

The International Medical Case Reports Journal is an international, peer-reviewed open-access journal publishing original case reports from all medical specialties. Previously unpublished medical posters are also accepted relating to any area of clinical or preclinica science. Submissions should not normally exceed 2,000 words or 4 published pages including figures, diagrams and references. The manuscript management system is completely online and includes a very quick and fair peer-review system, which is all easy to use. Visit http://www.dovepress.com/testimonials.php to read real quotes from published authors. 